\title{
Asymptomatic infection with Plasmodium falciparum and Plasmodium vivax in the Brazilian Amazon Basin: to treat or not to treat?
}

\author{
Mauro Shugiro Tada1, Ricardo de Godoi Mattos Ferreira², Tony Hiroshi Katsuragawa², \\ Rosimeire Cristina Dalla Martha', Joana D'Arc Neves Costa ${ }^{1}$, Letusa Albrecht ${ }^{3}$, \\ Gerhard Wunderlich ${ }^{3}$, Luiz Hildebrando Pereira da Silva²/+ \\ ${ }^{1}$ Centro de Pesquisa em Medicina Tropical ${ }^{2}$ Instituto de Pesquisa em Patologias Tropicais, Porto Velho, RO, Brasil \\ ${ }^{3}$ Departamento de Parasitologia, Universidade de São Paulo, São Paulo, SP, Brasil
}

In this study, we determined whether the treatment of asymptomatic parasites carriers (APCS), which are frequently found in the riverside localities of the Brazilian Amazon that are highly endemic for malaria, would decrease the local malaria incidence by decreasing the overall pool of parasites available to infect mosquitoes. In one village, the treatment of the 19 Plasmodium falciparum-infected APCs identified among the 270 residents led to a clear reduction $(Z=-2.39, p=0.017)$ in the incidence of clinical cases, suggesting that treatment of APCs is useful for controlling falciparum malaria. For vivax malaria, 120 APCs were identified among the 716 residents living in five villages. Comparing the monthly incidence of vivax malaria in two villages where the APCs were treated with the incidence in two villages where APCs were not treated yielded contradictory results and no clear differences in the incidence were observed $(Z=-0.09, p=0.933)$. Interestingly, a follow-up study showed that the frequency of clinical relapse in both the treated and untreated APCs was similar to the frequency seen in patients treated for primary clinical infections, thus indicating that vivax clinical immunity in the population is not species specific but only strain specific.

Key words: malaria - asymptomatic parasite carriers - Amazon

Asymptomatic infections by Plasmodium falciparum were first considered to be a consequence of naturally acquired immunity (NAI) (Christophers 1924) and were later designated as premonition (Sergent \& Parrot 1935). However, proof that NAI against the asexual blood stages of $P$. falciparum was of immune origin was provided by Cohen et al. (1961) only after the cure of an acutely infected child by the passive transfer of immunoglobulin obtained from asymptomatic Gambian adults. NAI was later described by Bruce-Chwatt (1980) as a common phenomenon in African adults living in holoendemic rural areas of the southern Sahara. Epidemiological studies in Gambia (Greenwood et al. 1987) have shown that death due to malaria occurs predominantly in infants and young children despite the presence of Plasmodium parasites in the blood of all age groups. Detailed studies conducted in Dielmo, Senegal (Trape et al. 1994), more precisely identified the characteristics of clinical (antidisease) immunity and anti-parasite immunity in holoendemic situations. First, clinical immunity to P. falciparum infection, which is principally observed in adults, protects against symptoms associated with malaria. Furthermore, this immunity may persist at high levels

Financial support: CNPq CT-Amazônia (575855/2008), FNS

(761/2006), Furnas Centrais Elétricas SA

+ Corresponding author: hildebrando@ipepatro.org.br

Received 17 October 2011

Accepted 2 February 2012 in clinically immune older children and adolescents independent of parasite density. Second, anti-parasite immunity develops progressively after adolescence and is characterised by a marked drop in the parasite density in infected young adults. Sterile immunity and total clinical protection were never observed, but the incidence of clinical infections was 40 times higher in children (0-4 years old) than in adults (Trape et al. 1994, Rogier et al. 1999a, b). The antibody-dependent anti-parasite immunity against asexual blood stages observed by Cohen et al. (1961) was later demonstrated not to be purely humoral, but also dependent upon the activity of a cellular component. In experimental primate infections (Faunder et al. 1984, Groux et al. 1990) and in human antibody transfer experiments (Bouharoum-Tavoun et al. 1990), the in vitro and in vivo anti-parasite antibody activities did not correlate and the in vivo activity was restricted to isotype-specific antibodies, specifically IgG1 and $\operatorname{IgG} 3$ in humans (Roussilhon et al. 2007) and cytophylic IgGs in Saimiri monkeys (Groux et al. 1990).

However, even though epidemiological studies in endemic areas have been performed, little is known regarding the clinical and anti-parasite NAI in infections with other human malaria parasites. Important and extensive studies (Collins \& Jeffery 1999, 2004) based on a retrospective examination of sporozoite and trophozoite-induced infections in patients with neurosyphilis who were treated with $P$. falciparum and Plasmodium vivax-based malaria therapy have provided some information concerning the development of immunity to $P$. vivax. These studies demonstrated that $P$. vivax-infected patients were more sensitive to re-infection with heter- 
ologous parasites than with $P$. falciparum, suggesting that at least in adults, NAI is more rapidly and efficiently acquired against $P$. falciparum.

Studies performed by our teams in Rondônia (RO), a state in the western Brazilian Amazon, showed that asymptomatic infection with $P$. vivax occurs frequently in the riverside areas of the Madeira River (Camargo et al. 1999). Detailed studies in the rural riverbank localities of the Madeira and Machado Rivers (Alves et al. 2002) showed that the overall prevalence of asymptomatic infection with $P$. falciparum or $P$. vivax was four-five times higher than the corresponding prevalence of symptomatic infection: $20-4.5 \%$ in a locality of the Madeira River and $49.5 \%$ and $10 \%$ in the localities of the Machado, respectively. In the latter localities, which consist of more dispersed and isolated human communities, equivalent frequencies of asymptomatic $P$. falciparum, $P$. vivax and mixed infections were observed $(16.2 \%, 18 \%$ and $15.3 \%$, respectively). Furthermore, we found that the development of NAI was related to the individual's age and length of residence in the endemic riverside area where the Anopheles darlingi vector is present at a high density (Gil et al. 2003, Cruz et al. 2009). Epidemiological surveys of the suburban and rural communities near the city of Porto Velho, the capital of RO (Fig. 1), also reported a relatively high prevalence of asymptomatic infections in the riverside areas (Gil et al. 2007, Tada et al. 2007, Katsuragawa et al. 2008, 2010). More detailed studies conducted in rural communities in the vicinity of the urban area of Porto Velho showed that asymptomatic infections were limited to long-term residents. By contrast, clinical malaria cases from the area were more frequent in nonresidents on short stays for fishing or tourism who slept in tents or shacks that rendered them more susceptible to Anopheles bites (Katsuragawa et al. 2010).

The demonstration that asymptomatic parasite carriers (APC) can infect anopheline vectors (Alves et al. 2005) and the discovery of asymptomatic infections in other areas of the Amazon (Ladeia-Andrade et al. 2009) raised the question of the role of APCs in malaria transmission. Consequently, treatment of APCs may be important for improving malaria control campaigns. In the present study, we present preliminary trials examining whether the treatment of APCs in highly endemic communities may decrease the incidence of clinical cases or affect other aspects of malaria transmission within the locality.

\section{SUBJECTS, MATERIALS AND METHODS}

Study area - The present study was performed in the following five suburban and rural riverbank localities of the Madeira River, situated to the south of the city of Porto Velho (Fig. 1) (all localities have been described in previous studies) (Gil et al. 2007, Tada et al. 2007, Katsuragawa et al. 2008, 2010): Vila Candelária (VC), Engenho Velho (EV), Santo Antônio (SA), Cachoeira do Teotônio (CT) and Vila Amazonas (VA). VC and EV are suburban, whereas CT and VA are rural localities approximately $30 \mathrm{~km}$ from the urban area. SA is at an intermediate distance from the urban area, with suburban and rural features. VC, SA and CT are accessible by road and regular bus service, but both EV and VA are situated on the left bank of the Madeira River and require boat transportation to access the right bank.

Population data for the five localities, which were provided by the municipality of Porto Velho, were extrapolated from data from the National Population Census in 2000 at the beginning of the study. A demographic census performed by our team updated the data. The total resident population of the five localities was 1,147 persons. A high incidence of malaria was officially recorded for the previous 10 years in the five localities by the Brazilian Health Ministry, with an annual parasite index of greater than 200 clinical cases per 1,000 inhabitants per year. In all of these localities, residents have access to malaria diagnosis and treatment in public health centres.

Study design - Preliminary definitions and data collection for the study design: APCs were identified via a positive microscopy result, a positive PCR result in the absence of malaria symptoms or by clinical signs up to 30 days following a positive result.

Clinical vivax malaria cases were classified as primary infections when they occurred in the follow-up population that previously tested negative for Plasmodium parasites by polymerase chain reaction (PCR). All of the clinical

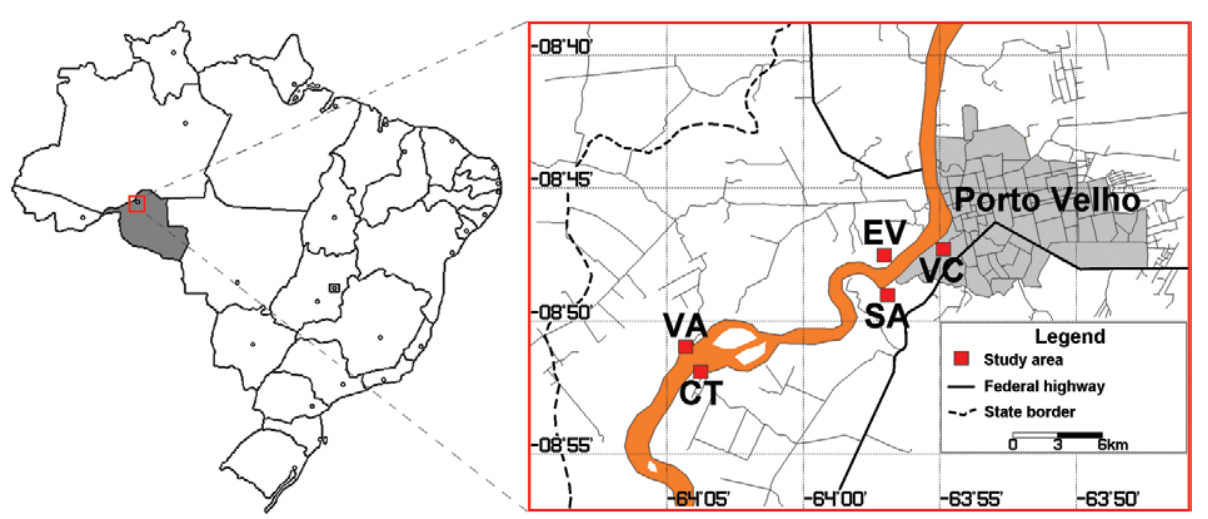

Fig. 1: study area location. The red quarters indicate the five riverine localities (CT: Cachoeira do Teotônio; EV: Engenho Velho; SA: Santo Antônio; VA: Vila Amazonas; VC: Vila Candelária). Madeira River is be indicated by orange colour; the green line indicates the federal highway. 
vivax malaria cases confirmed by microscopy within two-four month intervals and up to one year following the first treated and cured episode were considered to be relapses. Because there are no known markers to distinguish a vivax re-infection from a relapse (see Discussion), the adopted criteria were based on the previously identified probability of acquiring a vivax infection in one year in these localities (Tada et al. 2007, Katsuragawa et al. 2008,2010 ); based on these probabilities, a second infection is improbable and a third infection is unlikely.

Demographic and preliminary clinical surveys - All of the dwellings and respective residents of the five riverside communities were systematically identified. The dwellings were located using a Garmin eTrex Vista ${ }^{\mathrm{TM}}$ global positioning system device. The identity of all of the residents of each house, as well as their age, sex, socio-economic status and most important past health events, particularly those associated with malaria morbidity, were recorded. A brief physical and clinical examination was performed by a clinician, including the measurement of body temperature and the presentation of symptoms, such as liver or spleen enlargement.

Malaria baseline cross-sectional study - For the malaria prevalence survey and APC identification, a baseline cross-sectional survey was performed in each of the studied localities and $5 \mathrm{~mL}$ of venous blood was collected from all residents who were at least five years old for the diagnosis of malaria infection, irrespective of the presence of clinical symptoms.

Thick blood smears were Giemsa stained and examined at 1,000X magnification and at least 100 fields were examined by two experienced local technicians. All positive slides and a sample of slides collected from febrile individuals who were considered to be negative by the local technicians were re-examined by expert technicians at the Tropical Medicine Research Centre (CEPEM).

PCR was performed according to the method of Snounou (1996), with slight, previously described modifications (Tada et al. 2007, Katsuragawa et al. 2010). Once identified, the asymptomatic, PCR-positive patients were systematically monitored by health agents (see the section on malaria surveillance below) daily for 30 days before being considered an APC.

Treatment of APC - The present study consisted of two preliminary trials conducted to examine the effect of treatment of APCs on the transmission dynamics of malaria in a locality. In the first study, in $\mathrm{VC}$, one year of malaria surveillance (from April 2003-April 2004) was performed followed by a baseline cross-sectional study (April 2004) to identify all P. falciparum APCs. After the baseline cross-sectional study, all individuals that were PCR positive for P. falciparum were treated and one more year (from May 2005-May 2006) of malaria surveillance was performed. Detailed information about the malaria surveillance, the baseline cross-sectional study and the treatment protocol is provided in the next section. The data on the incidence of falciparum malaria during the surveillance periods before and after treatment of asymptomatic P. falciparum carriers were compared using the non-parametric Mann-Whitney $U$-test.
The second study was performed in a similar manner from 2006-2008 and extended to all five localities. At each locality, one year of malaria surveillance was performed followed by a baseline cross-sectional study to identify all asymptomatic Plasmodium carriers. After the baseline cross-sectional study, 119 APCs were identified in the five localities using PCR. All 66 residents of EV and SA that were PCR positive for $P$. vivax were treated, whereas the 53 PCR-positive residents from VC, $\mathrm{CT}$ and VA were maintained as controls and not treated. One additional year of malaria surveillance was performed in all five localities. Detailed information about the malaria surveillance, the baseline cross-sectional study and the treatment protocol is provided below.

The second study was designed to focus on both $P$. vivax and $P$. falciparum parasites. However, due to the reasons discussed below, only the $P$. vivax data could be confidently analysed. The data on the incidence of symptomatic malaria cases during the surveillance periods before and after the treatment procedures were compared using the non-parametric Mann-Whitney $U$-test.

Malaria surveillance - At all locations, malaria surveillance was conducted for at least two years: one year before the baseline cross-sectional study and one year after its completion. In both trials, the baseline survey was followed by one year of malaria surveillance, with visits to the dwellings at least five days per week. The inhabitants were interviewed about febrile episodes and every individual with a fever or any other symptoms potentially related to malaria was screened for the presence of malarial parasites. Furthermore, a clinical and parasitological examination was performed by our field team. Venous or finger-prick blood samples were obtained for the diagnosis of malaria via microscopy and PCR, as previously described (Tada et al. 2007, Katsuragawa et al. 2010). As part of the surveillance, the files of all patients were maintained at the two local outposts for malaria diagnosis. Thick blood smears were examined via microscopy by local technicians. All positive slides, a fraction of the negative slides and all of the negative slides from subjects presenting with fever or other potential malaria symptoms were reviewed by specialised technicians from CEPEM. Patients identified as presenting with symptomatic or asymptomatic $P$. vivax infections were closely monitored for possible relapses.

Malaria treatment - Patients presenting with clinical parasite infections were always treated according to the instructions in the Malaria Treatment Manual from the Brazilian Health Ministry (MS 2006). Quinine and doxycycline were used for the treatment of $P$. falciparum infections in the first preliminary trial. $P$. vivax infections were treated with chloroquine, followed by a short, seven-day course of primaquine (MS 2006). Treatment was initiated within $24-48 \mathrm{~h}$ of the initiation of symptoms. The same therapeutic scheme recommended by the MS was used for the treatment of all APCs. Primaquine was used for all symptomatic and asymptomatic carriers, with the exception of pregnant women and patients with a history of allergic or toxic reactions to the drug. 
Parasite identification, genotyping and microsatellite studies - The oligonucleotides and PCR conditions described by Snounou (1996) were used with slight modifications (Katsuragawa et al. 2010). Briefly, gDNA was amplified with primers specific for the ribosomal RNA genes and a second set of primers specific for $P$. falciparum, $P$. vivax or $P$. malariae. $P$. falciparum haplotypes were identified based on the size of a combination of 11 micro-satellite loci. The gDNA samples were amplified using a nested PCR protocol described by Anderson et al. (1999). In the second reaction, one Cy5-labelled primer was used, allowing for the semi-automated analysis of the marker sizes using an ALF2 (Amersham Pharmacia) automated sequencer. For the analysis of the size of the fragments, a molecular weight marker containing a 60 bp internal standard was added to each sample and 60bp, 97-bp, 175-bp and 219-bp length markers were used.

Ethical concerns - The project protocols were approved by the Ethical Research Committee of the CEPEM (CEP-CEPEM) of the Rondônia State Health Bureau and considered "scientifically and technically in accordance with ethical principles of conduct". Haematological and serological samples from individuals in the study population were collected after informed consent and written agreements were obtained from each individual (CEP-CEPEM decision 25/2004).

\section{RESULTS}

First preliminary survey - Effects of the treatment of asymptomatic carriers on the incidence of falciparum malaria in VC. The first trial was performed in VC from April 2003-April 2005. The incidence of malaria in this locality was investigated by CEPEM- Research Institute In Tropical Pathologists epidemiologists. A demographic survey performed in March 2003 reported 270 residents. The baseline survey for the identification of P. falciparum infections performed in March 2004 with 223 residents who were at least five years old $(82.7 \%)$ identified 16 P. falciparum APCs, 32 P. vivax APCs and three APCs with mixed infections. The 19 patients with $P$. falciparum infections were treated in May. The malaria surveillance follow-up was maintained from May
2003-April 2005 and is summarised in Fig. 2, which shows the monthly distribution of clinical falciparum malaria cases during the surveillance period.

As shown in Fig. 2, the number of clinical cases of falciparum malaria decreased significantly in the months following the treatment of the APCs in May 2004 and no $P$. falciparum episodes were recorded from May-September $(Z=-2.39, \mathrm{p}=0.017)$. The only falciparum malaria case in October 2004 was an adult who arrived in VC with a clinical infection that had been diagnosed as falciparum malaria in another locality of RO. The previous microscopic examination of the thick smear performed on September 19 revealed the presence of $P$. falciparum gametocytes, but primaquine treatment could not be administered because of medical recommendations. In addition, four other $P$. falciparum cases occurred from November 2004-January 2005 that affected patients living in residences near that of the imported case (2 in November 2004, 1 in December 2004 and 1 in January 2005). The genotypes of the parasites collected from the four clinical cases, as determined using microsatellite analysis (Table I), were identical to that of the imported case and showed allelic differences from the consensus genotype of parasites observed in VC from 2003-2004. Three alleles in these four patients had not been previously found in this locality (Table I).

Second preliminary trial - Effects of the treatment of asymptomatic $P$. vivax carriers on the incidence of vivax malaria. This trial was initially planned for both $P$. falciparum and P. vivax APCs between 2006-2007. However, in mid-2007, the MS detected a clear decrease in the incidence of falciparum malaria throughout the endemic area of the Brazilian Amazon (SIVEP-Malária 2007) that coincided with the changes in treatment schedules adopted by the MS. Consequently, the study on P. falciparum APCs was cancelled and the project was restricted to the effects of treating $P$. vivax APCs. Preliminary demographic surveys were developed in 2006 in the five localities situated in the suburban and rural areas near the southern limit of Porto Velho (VC, EV, SA, CT and VA) (Fig. 1). The corresponding baseline survey performed to identify APCs was conducted

P. falciparum cases at Vila Candelaria

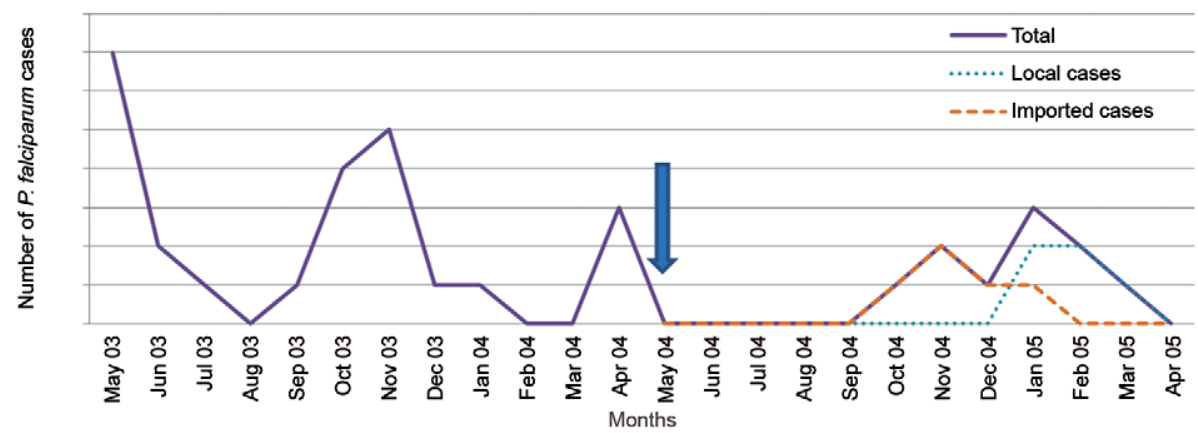

Fig, 2: Plasmodium falciparum cases at Vila Candelária. The malaria surveillance follow-up from September 2005-September 2007. The arrow indicates the treatment application. 
progressively from July-November 2006 in the localities shown in Table II. PCR analysis of blood samples identified 119 P. vivax APCs among the 716 participants in the five localities (16.8\%). Chloroquine/primaquine combination therapy was administered to 66 APC patients, 35 from EV and 31 from SA, 30 days after the follow-up that confirmed an absence of symptoms in the patients. No treatment was administered to the other 53 APCs (23 from VC, 18 from CT and 12 from VA). Malaria surveillance was maintained for both groups for the following year. As shown in Fig. 3, no decrease in the incidence of $P$. vivax malaria was observed in $\mathrm{EV}$ in the months following the treatment of APCs in August 2006 ( $Z$ $=-0.30, \mathrm{p}=0.762$ ), whereas in SA, a 2.25 -fold increase in the mean number of cases was observed $(Z=-2.84$, p $=0.005)$. However, it must be emphasised that the increase was dependent upon data from the end of the observation period (see Discussion). In the control localities where the APCs were not treated, no decrease in the incidence was observed in CT $(Z=-0.55, \mathrm{p}=0.585)$, whereas in VA, a significant decrease was observed $(Z$ $=-2.44, p=0.015$ ) (see Discussion). The follow-up of ma- laria cases in VC suffered from a discontinuity of more than three months due to a failure of a health agent responsible for the task and the difficulties in reorganising the surveillance team led us to eliminate $\mathrm{VC}$ from follow-up. These contradictory results do not allow for elucidation of the effect of treatment of vivax APCs (see Discussion).

Second preliminary trial - Effects of treatment of asymptomatic $P$. vivax carriers on the frequency of vivax malaria relapse. The comparison of the frequency of relapse in APC-treated localities (EV and SA) and untreated localities (VC, CT and VA) provided some unexpected results (Tables III, IV). However, there was a discontinuity in the follow-up of the malaria cases in $\mathrm{VC}$ and the data could not be used, as explained in the previous section. However, because all clinical cases were diagnosed and treated, a relapse register was maintained in VC. Both treated and untreated vivax APCs presented with an equal number of relapses, which were characterised by the presence of clinical symptoms and a positive microscopic examination during the 12 months of post-intervention surveillance. As seen in Table III, 21 of the 66 patients

TABLE I

Comparison of micro-satellite allele sizes of Vila Candelaria's consensus and new Plasmodium falciparum cases

\begin{tabular}{lcccc}
\hline Allele & In the four new cases & Different alleles found in previous cases & Alleles not found before & Alleles(n) \\
\hline TAI & 174 & $168,171,174,177,180,183$ & - & 6 \\
Poly-a & 182 & $149,152,155,170,176,182,185,191$ & 87 & 8 \\
TAA60 & 87 & $75,78,84,93$ & - & 5 \\
ARA2 & 66 & 66 & - & 1 \\
Pfg377 & 102 & 96,99 & - & 3 \\
PfPK2 & 174 & $162,171,177$ & - & 3 \\
TAA87 & 114 & $93,96,99,111,114$ & - & 4 \\
TAA109 & 163 & $163,166,175$ & - & 3 \\
TAA81 & 121 & $115,118,121,124,127,139$ & 90 \\
TAA42 & 188 & $188,194,197,203$ & 4 \\
2490 & 87,90 & $84,87,93$ & 4 \\
\hline
\end{tabular}

TABLE II

Asymptomatic malaria carrier's prevalence in riverine localities of the Madeira River

\begin{tabular}{|c|c|c|c|c|c|c|c|c|c|c|}
\hline \multirow[b]{2}{*}{ Locality } & \multirow{2}{*}{$\begin{array}{c}\text { Baseline cross section } \\
\text { (2006) }\end{array}$} & \multirow[b]{2}{*}{ Population } & \multicolumn{4}{|c|}{ Thick smear } & \multicolumn{4}{|c|}{ PCR } \\
\hline & & & $\mathrm{n}$ & $\mathrm{Pv}^{+}$ & $\mathrm{Pf}+$ & Mixed $+^{a}$ & $\mathrm{n}$ & $\mathrm{Pv}^{+}$ & $\mathrm{Pf}+$ & Mixed + \\
\hline Engenho Velho & July & 139 & 121 & 1 & - & - & 118 & 35 & 7 & 5 \\
\hline Santo Antônio & August & 251 & 194 & 2 & 1 & - & 199 & 31 & 5 & 1 \\
\hline Vila Candelária & September & 268 & 205 & - & 1 & - & 205 & 23 & 3 & - \\
\hline Cachoeira do Teotônio & November & 343 & 119 & 4 & - & - & 119 & 18 & - & - \\
\hline Vila Amazonas & November & 144 & 77 & - & 1 & - & 77 & 12 & 2 & - \\
\hline Total & - & 1,147 & 716 & 7 & 3 & - & 718 & 119 & 17 & 6 \\
\hline
\end{tabular}

a: Plasmodium vivax (Pv) and Plasmodium falciparum (Pf); PCR: polymerase chain reaction. 
(31.8\%) from EV and SA that were treated for asymptomatic infections at time zero $\left(\mathrm{T}^{0}\right)$ presented with relapses $\left(\mathrm{R}^{1}+\mathrm{R}^{2}\right)$. This incidence of relapse was somewhat, but not significantly, greater than the incidence of relapse in the untreated APCs in VC, CT and VA [16 of 53 (30.2\%), $\chi^{2}=0.134,1$ degree of freedom, $p=0.843$. Additionally, the difference in the overall number of clinical infections in the treated and untreated localities was not statistically significant $(Z=-0.085, \mathrm{p}=0.933)$. Furthermore, over the same period, the frequency of relapse in patients presenting as PCR negative at $\mathrm{T}^{0}$ and later presenting with a clinical infection that was characterised as a primary vivax infection in the five localities (27 in 89, 27\%) was equal. These values fall within the range of relapse frequencies both reported in the literature following short-term primaquine treatment (Villalobos-Salcedo etal. 2000) and observed in patients treated for clinical $P$. vivax infections residing in other localities of the study. In addition, the delay (in months) observed between primary infection and relapse was equivalent in the treated and untreated asymptomatic infections (Table IV). Taken together, these observations raise questions about the nature of NAI in $P$. vivax infections. It is important to note that the malaria surveillance revealed that 14 of the 51 untreated vivax APCs from VC, CT and VA presented with clinical episodes with positive microscopy examinations that were classified as relapses using the criteria adopted in this study (see Subjects, Materials and Methods).

\section{DISCUSSION}

The present study aimed to address whether treatment of malaria APCs in an endemic area would decrease malaria transmission within the community. The results reported here clearly illustrate the difficulties in drawing conclusions from field studies. The difficulties in this case derive from the following independent and complex variables that contributed to or interfered with the results: (i) the nature of the protective clinical and anti-parasite immunity of the APCs and the distribution of the APCs in the community, (ii) parasite variability and the mechanisms used by the parasite to escape from the host immune response, (iii) the susceptibility of local parasite populations to the available anti-malarial drugs, (iv) the transmission capacity, seasonal density variations and efficiency of the local malaria vector, (v) the physical and climatic factors involved in the proliferation of the vector and (vi) demographic instability, which is particularly high in Brazilian Amazon communities.

Despite these difficulties, the results of the first trial clearly indicate a favourable effect of treating falciparum APCs because a significant decrease was observed in the local incidence of falciparum malaria in the months following intervention. The new falciparum malaria cases that appeared seven months after the intervention were all imported. The evaluation of the positive effect of decreasing the incidence was facilitated by the fact that the APCs were treated when no clinical cases were observed in the locality, indicating that the local parasite pool was only represented by the parasites circulating in the APCs. At the same time, our results show the important role of imported malaria in maintaining endemicity in a given locality. As described in the Results section, microsatellite analysis (dalla-Martha et al. 2007) demonstrated that following treatment of the APCs, the few falciparum malaria cases that were observed among the residents of the locality were due to the same imported parasite lineage. Therefore, we conclude that the benefits of treating APCs also require the rigorous control of imported malaria, which is not easily implemented in poor communities.

The evaluation of the effect of the treatment of $P$. vivax APCs provided contradictory results. Comparing the incidences in the 12 months preceding and following treatment in two villages (EV and SA) with the incidences in two control villages (CT and VA) where the APCs were not treated, we detected no variation in the incidences in both EV (treated APCs) and VA (untreat-

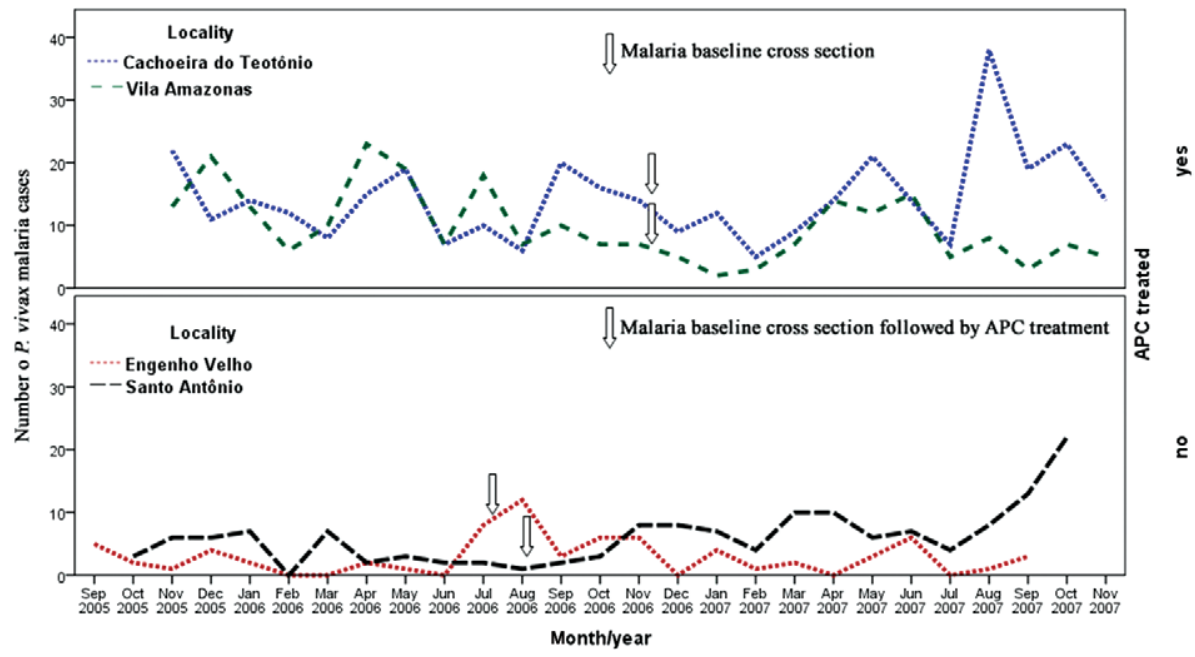

Fig. 3: incidence of Plasmodium vivax malaria in the study localities from September 2005-September 2007. APC: asymptomatic parasite carriers (APC). 


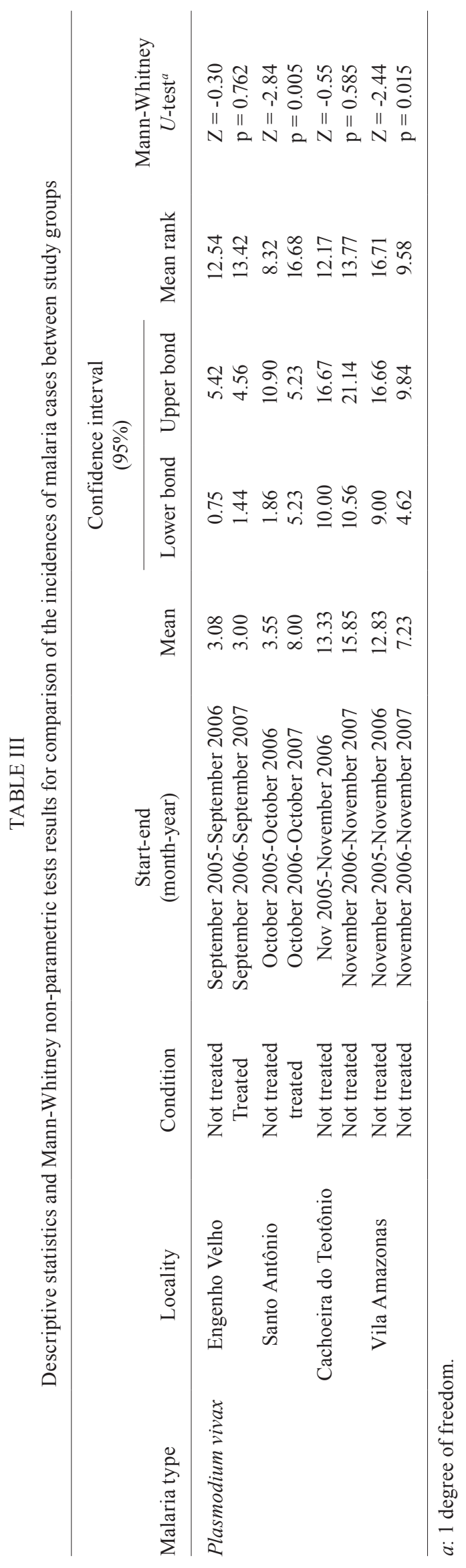

ed APCs). By contrast, the incidence increased in SA (treated APCs) and decreased in VA (untreated APCs). It must be noted that in the months following the intervention, the SA village experienced demographic instability related to the preparation of the Santo Antônio hydroelectric power plant construction site. Migration, including the presence of hydroelectric company employees, occurred after June 2007 and this migration may have influenced the malaria incidence. Furthermore, in contrast with the first preliminary survey, in which the intervention took place when no clinical falciparum malaria cases occurred, in the second preliminary survey for $P$. vivax, the treatment of the APCs was performed when there was a high incidence of clinical vivax infections (Fig. 3). Therefore, the malaria surveillance methodology used before and after the APC treatment to measure the effects on malaria transmission cannot provide a conclusive answer in the context of a highly endemic situation, whereas it was a useful method to measure transmission in lowly endemic situations (e.g., falciparum infection).

By contrast, in the surveillance of vivax malaria in the second preliminary survey, the follow-up of relapses in conjunction with APC treatment provided important information. However, a question that needs to be clarified concerns the criteria adopted to define transmission dynamics by measuring relapses. In this trial, we considered primary $P$. vivax episodes to be infections affecting patients that were PCR negative in the baseline survey, but presented with clinical infections later. The subsequent clinical vivax malaria episodes that occurred in the same patient in the 12 months following the treatment and cure of the primary clinical infection were considered relapses. Of course, re-infections could not be excluded because the risk of a vivax infection in this area is nearly $15 \%$ in one year (Tada et al. 2007). However, if one re-infection within one year in the same subject is statistically possible, two-three subsequent re-infections are unlikely to occur. Using the same procedures and criteria for patients treated for asymptomatic vivax malaria infections, clinical infections occurring in the 12 months following the APC treatment were also considered relapses.

Using these criteria, some unexpected results were observed. Whereas primary clinical $P$. vivax infections were followed by frequent relapses, as has been described in studies performed in the same region (VillalobosSalcedo et al. 2000), surprisingly, in the 12 months following the intervention, both the treated and untreated APCs presented with one-three relapses at an equivalent frequency. It is important to note that relapses were only considered to be clinical infections following parasite confirmation using microscopy, which eliminates the possibility of recrudescence (Table IV).

In either case, even if the repetitive clinical episodes in the same patients are re-infections and not relapses, the clinical infections observed in the treated and untreated vivax APCs raise questions concerning the development of naturally acquired protective anti-parasite immunity in $P$. vivax infections. Distinguishing $P$. vivax re-infections from relapses in an active endemic area is difficult because of the polyclonal nature of the liver 
TABLE IV

Relapses frequency in both asymptomatic and symptomatic vivax malaria patients

\begin{tabular}{|c|c|c|c|c|c|c|c|c|c|}
\hline \multirow[b]{2}{*}{ Locality } & \multirow[b]{2}{*}{$\begin{array}{c}\mathrm{APC} \\
\text { treated }\end{array}$} & \multicolumn{4}{|c|}{$\mathrm{A}-\mathrm{PCR}(+)$} & \multicolumn{4}{|c|}{ P-PCR(-) } \\
\hline & & $\begin{array}{c}\text { PCR+ } \\
\text { (n) }\end{array}$ & $\mathrm{R}^{1}$ & $\mathrm{R}^{2}$ & $\mathrm{R}^{3 /+}$ & $\begin{array}{c}\text { PCR- } \\
\text { (n) }\end{array}$ & I & $\mathrm{R}^{1}$ & $\mathrm{R}^{2 /+}$ \\
\hline Engenho Velho ${ }^{a}$ & Yes & 35 & 11 & 3 & - & 71 & 7 & 4 & - \\
\hline Santo Antônio ${ }^{b}$ & Yes & 31 & 5 & 2 & 1 & 162 & 35 & 4 & 3 \\
\hline Vila Candelária $^{c}$ & No & 23 & 2 & 3 & - & 179 & 25 & 3 & 5 \\
\hline Cachoeira do Teotônio $^{d}$ & No & 18 & 4 & 2 & - & 101 & 13 & 5 & - \\
\hline Vila Amazonas ${ }^{e}$ & No & 12 & 2 & 1 & 2 & 63 & 9 & - & 1 \\
\hline Total & - & 119 & 24 & 11 & 3 & 576 & 89 & 16 & 9 \\
\hline
\end{tabular}

$a$ : follow up conducted from September 2006-September 2007; $b$ : follow up conducted from October 2006-October 2007; $c$ : follow up conducted from September 2006-September 2007; $d$ : follow up conducted from November 2006-November 2007; $e$ : follow up conducted from November 2006-November 2007; APC: asymptomatic parasite carriers; A-PCR(+): asymptomatic Plasmodium vivax patients polymerase chain reaction $(\mathrm{PCR})(+)$ at malaria baseline cross section; I: new infections considered prime infections; P-PCR(-): patients with clinical vivax infection that were $\mathrm{PCR}(-)$ at baseline cross section; $\mathrm{R}^{1}, \mathrm{R}^{2}, \mathrm{R}^{2 /+}, \mathrm{R}^{3 /+}$ : relapses (always symptomatic infections; the exponents indicate number of relapses).

hypnozoites originating from the polyclonal sporozoite population introduced by the mosquito (Druilhe et al. 1996, Kirchgatter \& Del Portillo 1998).

The mechanisms involved in protective immunity, either clinical or anti-parasite, remain poorly understood for vivax malaria. Antibody-dependent immune responses are considered the basic mechanism of anti-parasite immunity in P. falciparum infections (Cohen et al. 1961, Fandeur et al. 1984, Bouharoum-Tavoun et al. 1990, Groux et al. 1990, Gysin et al. 1992). However, no equivalent observations are available for $P$. vivax and how anti-parasite immunity develops against $P$. vivax remains poorly understood. Our results are in agreement with those of Collins and Jeffery (1999), who reported on the retrospective examination of sporozoite and trophozoite-induced infections in neurosyphilitic patients treated with vivax malaria therapy (Camargo et al. 1999, Alves et al. 2002). They found that the development of anti-parasite immunity is variant specific. In conclusion, if the treatment of $P$. vivax APCs is proposed with the aim of interfering with $P$. vivax transmission by reducing the pool of parasites, the results of the present study indicate that this intervention would likely have no practical value. As APCs infect the vector less efficiently (Alves et al. 2005), but present with frequent symptomatic relapses or re-infections with higher parasitaemia, treatment of APCs would have minor consequences for malaria transmission.

Molecular tests are not widely used in the diagnosis of malaria in the general public. However, in the present study and in eventual future studies, a preliminary survey by PCR was or should be performed only once at the beginning of the intervention followed by surveillance using microscopy for diagnosis. In eradication programs, the procedure could be performed in nearby localities, individually. The role of APCs in malaria transmission has been and remains the subject of discussion by experts worldwide and there is still no consensus. In the present study, we conclude that there is not one single answer to the question. Our results show that the treatment of falciparum APCs has a positive effect on decreasing falciparum malaria transmission in one locality. However, this was observed in a locality with a relatively low prevalence of falciparum malaria and also depended on strong surveillance to prevent re-introduction of imported malaria by visitors and/or the arrival of new residents harbouring the parasite. With respect to vivax malaria, our results show that when using a short course of primaquine, the frequency of relapse is high and therefore, the treatment of vivax APCs would have practically no effect on malaria transmission. In addition, is not possible to use a 14-day primaquine treatment on vivax APCs, which does not completely eliminate relapses (Villalobos-Salcedo et al. 2000). Therefore, we conclude that the treatment of vivax APCs is not useful. However, if new drugs are discovered with activity against hypnozoites or if other methods to avoid relapses are developed, the treatment of vivax APCs may be useful.

\section{ACKNOWLEDGEMENTS}

To Gordon Langsley, for reviewing the manuscript, and Cor Jesus Fernandes Fontes, for useful suggestions.

\section{REFERENCES}

Alves FP, Durlacher RR, Menezes MJ, Krieger H, Pereira-da-Silva LH, Camargo EP 2002. High prevalence of asymptomatic Plasmodium vivax and Plasmodium falciparum infections in native Amazonian populations. Am J Trop Med Hyg 66: 641-648.

Alves FP, Gil LH, Marrelli MT, Ribolla PE, Camargo EP, Pereirada-Silva LH 2005. Asymptomatic carriers of Plasmodium spp as infection source for malaria vector mosquitoes in the Brazilian Amazon. J Med Entomol 42: 777-779.

Anderson TJ, Su XZ, Bockarie M, Lagog M, Day KP 1999. Twelve microsatellite markers for characterization of Plasmodium falciparum from finger-prick blood samples. Parasitology 119: 113-125. 
Bouharoum-Tayoun H, Attanath P, Sabchareon A, Chonguphajaisidhi T, Druilhe P 1990. Antibodies that protect humans against Plasmodium falciparum blood stages do not on their own inhibit parasite growth and invasion in vitro, but act in cooperation with monocytes. J Exp Med 172: 1633-1641.

Bruce-Chwatt LJ 1980. Essential malariology, Willian Heinemann Medical Books, London, 354 pp.

Camargo EP, Alves FP, Pereira-da-Silva LH 1999. Symptomless Plasmodium vivax infections in native Amazonians. Lancet 353: 1415-116.

Christophers SR 1924. The mechanism of immunity against malaria communities living under hyperendemic conditions. Indian $J$ Med Res 12: 273-294.

Cohen S, McGregor IA, Carrington S 1961. Gamma globulin and acquired immunity to human malaria. Nature 192: 733-737.

Collins WE, Jeffery GM 1999. Retrospective examination of a secondary sporozoite and trophozoite induced infections with Plasmodium falciparum: development of parasitological and clinical immunity following secondary infection. Am J Trop Med Hyg 6: 20-35.

Collins WE, Jeffery GM 2004. A retrospective examination of reinfection of humans with Plasmodium vivax. Am J Trop Med Hyg 70: 642-644.

Cruz RM, Gil LH, de Almeida e Silva A, da Silva Araujo M, Katsuragawa TH 2009. Mosquito abundance and behaviour in the influence area of the hydroelectric complex on the Madeira River, western Amazon, Brazil. Trans R Soc Trop Med Hyg 103: 1174-1176.

dalla Martha RC, Tada MS, Ferreira RG de M, da Silva LHP, Wunderlich G 2007. Microsatellite characterization of Plasmodium falciparum from symptomatic and non-symptomatic infections from the western Amazon reveals the existence of non-symptomatic infection-associated genotype. Mem Inst Oswaldo Cruz 102: 293-298.

Druilhe P, Daubersies P, Patarapotikul J, Gentil C, Chene L, Chongsuphajaisiddhi T, Mellouk S, Langsley G 1996. A primary malarial infection is composed of a very wide range of genetically diverse but related parasites. J Clin Invest 101: 2008-2016.

Fandeur T, Dubois P, Gysyn J, Dedet JP, Pereira da Silva LH 1984. In vivo and in vitro studies on protective antibodies against Plasmodium falciparum in the squirrel monkey. $J$ Immunol 132: 432-437.

Gil LH, Alves FP, Zieler H, Salcedo JM, Durlacher RR, Cunha RPA, Tada MS, Camargo LMA, Camargo EP, Pereira-da-Silva LH 2003. Seasonal malaria transmission and variation of anopheline density in two distinct endemic areas in Brazilian Amazonia. J Med Entomol 40: 636-641.

Gil LHS, Tada MS, Katsuragawa TH, Ribolla PEM, da Silva LHP 2007. Urban and suburban malaria in Rondônia (Brazilian western Amazon). II: Perennial transmissions with high anopheline densities are associated with human environmental changes. Mem Inst Oswaldo Cruz 102: 271-276.

Greenwood BM, Bradley EK, Greenwood AM, Byass P, Jammeh K, Marsh K, Tulloch S, Oldfield FS, Hayes R 1987. Mortality and morbidity from malaria among children in a rural area of the Gambia, west Africa. Trans R Soc Trop Med Hyg 81: 478-486.

Groux H Perraut R, Garraud O, Poingt JP, Gysin J 1990. Functional characterization of the antibody mediated protection against blood stages of Plasmodium falciparum in the monkey Saimiri sciureus. Eur J Immunol 20: 2317-2323.
Gysin J, Druilhe P, da Silva LP 1992. Mechanisms of immune protection in the asexual blood stage infection by Plasmodium falciparum: analysis by in vitro and ex-vivo assays. Mem Inst $\mathrm{Os}_{\mathrm{s}}$ waldo Cruz 87 (Suppl. V): 145-149.

Katsuragawa TH, Gil LHS, Tada MS, Pereira da Silva LH 2008. Endemic and epidemic diseases in Amazonia: malaria and other emerging diseases in the riverine areas of the Madeira River. A school case. Estud Av 22: 112-141.

Katsuragawa TH, Gil LHS, Tada MS, Silva AA, Costa JDN, Araújo MS, Escobar AL, Pereira-da-Silva LH 2010. The dynamics of transmission and spatial distribution of malaria in riverside areas of Porto Velho, Rondônia, in the Amazon Region of Brazil. PLoS ONE 5: e9245.

Kirchgatter K, Del Portillo HA 1998. Molecular analysis of Plasmodium vivax relapses using the MSP1 molecule as a genetic marker. J Infect Dis 177: 511-515.

Ladeia-Andrade S, Ferreira MU, de Carvalho ME, Curado I, Coura JR 2009. Age-dependent acquisition of protective immunity to malaria in riverine populations of the Amazon Basin of Brazil. Am J Trop Med Hyg 80: 452-459.

MS - Ministério da Saúde 2006. Ações de controle da malária. Manual para profissionais da saúde na atenção básica, MS, Brasília, 52 pp.

Rogier C, Ly AB, Tall A, Cisse B, Trape JF 1999a. Plasmodium falciparum clinical malaria in Dielmo, a holoendemic area in Senegal: no influence of acquired immunity on initial symptomatology and severity of malaria attacks. Am J Trop Med Hyg 60: 410-420.

Rogier C, Tall A, Diagne N, Fontenille D, Spiegel A, Trape JF 1999b. Plasmodium falciparum clinical malaria: lessons from longitudinal studies in Senegal. Parassitologia 41: 255-259.

Roussilhon C, Oeuvray C, Müler-Graf C, Tall A, Roger C, Trape JF, Theisen M, Balde A, Pérignon JL, Druilhe P 2007. Long term clinical protection from falciparum malaria is strongly associated with IgG3 antibodies to surface protein 3. PLoS Med 4: e320.

Sergent E, Parrot L 1935. L'immunite la premonition et la resitance innee. Arch Inst Pasteur Algerie 13: 279-319.

SIVEP-Malária - Sistema de Informação de Vigilância Epidemiológica 2007. Epidemiologic surveillance of information system. Available from: saude.gov.br/sivep_malaria.

Snounou G 1996. Detection and identification of the four malaria parasite species infecting humans by PCR amplification. Methods Mol Biol 50: 263-291.

Tada MS, Marques RP, Mesquita E, Dalla Martha RC, Rodrigues JA, Costa JDN, Pepelascov RR, Katsuragawa TH, Pereira-da-Silva LH 2007. Urban malaria in the Brazilan western Amazon Region. I. High prevalence of asymptomatic carriers in an urban riverside district is associated with a high level of clinical malaria. Mem Inst Oswaldo Cruz 102: 263-269.

Trape JF, Rogier C, Konate L, Diagne N, Bouganali H, Cangue B, Legros F, Badji A, Ndiaye G, Ndiaye P, Brahimi K, Faye O, Druilhe P, Pereira-da-Silva L 1994. The Dielmo project: a longitudinal study of natural malaria infection and the mechanisms of protective immunity in a community living in a holoendemic area. Am J Trop Med Hyg 51: 123-137.

Villalobos-Salcedo JM, Tada MS, Kimura E, Menezes MJ, Pereira da Silva LH 2000. In vivo sensitivity of Plasmodium vivax isolates from Rondônia (western Amazon region, Brazil) to regimens including chloroquine. Ann Trop Med Parasitol 94: 749-58. 